\title{
Impact of leg bending in the patient-specific computational fluid dynamics of popliteal stenting
}

\author{
A. Ferrarini ${ }^{1} \cdot$ A. Finotello ${ }^{2} \cdot$ G. Salsano ${ }^{3} \cdot$ F. Auricchio ${ }^{1} \cdot$ D. Palombo ${ }^{4} \cdot$ G. Spinella ${ }^{4} \cdot$ B. Pane ${ }^{4} \cdot$ M. Conti $^{1}$
}

Received: 23 December 2020 / Revised: 17 January 2021 / Accepted: 5 February 2021 / Published online: 30 March 2021

(C) The Author(s) 2021

\begin{abstract}
Endovascular treatment of the femoro-popliteal artery has recently become a valuable therapeutic option for popliteal arterial aneurysms. However, its efficacy remains controversial due to the relatively high rate of complications, such as stent occlusion as result of intra-stent thrombosis. The elucidation of the interplay among vessel geometrical features, local hemodynamics, and leg bending seems crucial to understand onset and progression of popliteal intra-stent thrombosis. To this aim, patientspecific computational fluid dynamic simulations were performed in order to assess the intra-stent hemodynamics of two patients endovascularly treated for popliteal arterial aneurysm by stent-grafts and experiencing intra-stent thrombosis. Both Newtonian and non-Newtonian blood rheological models were considered. Results were presented in terms of tortuosity, luminal area exposed to low $(<0.4 \mathrm{~Pa})$ and high $(>1.5 \mathrm{~Pa})$ time-averaged wall shear stress (TAWSS), area exposed to high $(>0.3)$ oscillatory shear index (OSI), and flow helicity. Study outcomes demonstrated that leg bending induced significant hemodynamic differences ( $>50 \%$ increase) in both patients for all the considered variables, except for OSI in one of the two considered patients. In both leg configurations, stent-graft overlapping induced a severe discontinuity of the lumen diameter where the proximal stented zone is characterized by low tortuosity, low velocity, low helicity, low TAWSS, and high OSI; while the distal part has higher tortuosity, velocity, helicity, TAWSS, and lower OSI. Sensitivity study on applied boundary conditions showed that the different inlet velocity profiles for a given inlet waveform affect slightly the numerical solution; conversely, the shape and magnitude of the prescribed inlet waveform is determinant. Focusing on the comparison between the Newtonian and non-Newtonian blood models, the area with low TAWSS is greater in the Newtonian model for both patients, while no significant difference occurs between the surfaces with high TAWSS.
\end{abstract}

Keywords Popliteal artery aneurysm $\cdot$ Peripheral stenting $\cdot$ Endovascular treatment $\cdot$ Femoropopliteal segment $\cdot$ Medical image analysis

\section{Introduction}

Executive Editor: Jizeng Wang

Supplementary Information The online version contains supplementary material available at https://doi.org/10.1007/s10409021-01066-2.

M. Conti

michele.conti@unipv.it

1 Department of Civil Engineering and Architecture, University of Pavia, Pavia, Italy

2 Department of Surgical and Integrated Diagnostic Sciences, University of Genoa, Genoa, Italy

3 Department of Radiology, IRCCS Ospedale Policlinico San Martino, Genoa, Italy

4 Vascular and Endovascular Surgery Unit, IRCCS Ospedale Policlinico San Martino, Genoa, Italy
Popliteal arterial aneurysms (PAA) are common peripheral aneurysms. Although in the last few years endovascular treatment of the femoro-popliteal artery (FPA) has become a valuable therapeutic option, its efficacy remains controversial due to the relatively high rate of complications, such as stent occlusion, intra-stent thrombosis or even stent fracture [1]. All these drawbacks could be related to the intrinsic morphology of the FPA segment that presents unique characteristics in terms of extreme mobility and biomechanical forces and severe loading conditions due to repetitive leg flexion during daily activities [2]. If on the one hand the stent fracture can be traced back almost exclusively to repeated bending of the stented leg, on the other the mechanisms that lead to intra-stent thrombosis are not fully understood even 
if hemodynamics is suspected of playing an important role in this process [3].

Computational fluid dynamics (CFD) analyses are increasingly exploited to quantify the blood flow inside the FPA and evaluate the changes on hemodynamic patterns due to the combination of endovascular stenting and leg movements. Blood flow patterns, and in particular low shear stress, prominent secondary flows or huge variations of arterial wall shear stress (WSS) are indeed known to correlate with pathological conditions [4-6], as briefly resumed in the following.

First studies investigating flow patterns in patient-specific superficial femoral arteries date back to 2006 by Wood et al. [6], who combined magnetic resonance imaging and CFD to assess the relationship between curvature and tortuosity of superficial femoral arteries and flow patterns as function of sex and age. More recently, the study of Desyatova et al. [7], who investigated the effects of aging on mechanical stresses, deformations, and hemodynamics, has identified the popliteal artery as the location with greatest intramural stresses along the leg arteries. Moreover, the association of vessel restenosis with hemodynamical markers derived from blood flow has been investigated by Gogkol et al. [3], in patients undergoing endovascular treatment for peripheral artery diseases (PAD). However, the proposed work was based on vessel geometries reconstructed from 2D angiographic images thus idealizing the lumen cross-sections. This limitation was overcome by Colombo et al. [8], who presented a fully patient-specific computational framework based on geometric reconstructions from Computed Tomography (CT) images and boundary conditions taken from Doppler ultrasound images. However, despite the proposed innovations, only the straight-leg configuration has been studied, thus neglecting the analysis of the effects of leg bending on the geometry and hemodynamics of the stented area. In a more recent study led by Colombo et al. [9], knee flexion and complete movement of walking have been assessed in an idealized model of FPA. Finally, the impact of leg bending on geometrical and hemodynamic features have been investigated in our previous work, where patient-specific CFD simulations have been performed on a single patient, using literature boundary conditions [10] and Newtonian model for blood rheology. Although it is known that blood is a nonNewtonian fluid [11], literature review about CFD modeling of the actual rheology of blood is controversial. While the assumption of treating blood as a Newtonian fluid is widely accepted [12], it still represents a pivotal issue in case of small or mid arteries. Moreover, while some studies highlighted the importance of the non-Newtonian rheology [13], others found that the use of a Newtonian blood model represents a good approximation [14,15]. In particular, focusing on modeling of the FPA, most of the studies $[3,6,16]$ assumed a constant viscosity, even if Colombo et al. [8] adopted the
Carreau model to describe the non-Newtonian viscosity of blood.

According to the literature, CFD provides a useful tool for understanding and predicting disease progression and hemodynamic-related post-stent complications. However, literature studies about patient specific CFD of popliteal stenting are scarce; in particular, many of them involve idealized geometry [3,9] and literature boundary conditions $[3,10]$, without any information regarding the follow-up intra-stent thrombosis. Moreover, morphological variations during knee flexion in the FPA could significantly influence the local hemodynamic $[9,10]$. To the best of our knowledge, a complete computational study including all these aspects is still missing.

Based on such considerations, we performed patientspecific CFD simulations in order to assess the impact of leg bending and the interplay among geometrical features on the local hemodynamic of two patients treated with endovascular stent-graft placement for PAA, experiencing intra-stent thrombosis. Moreover, we deepened and improved our previous study [10] by investigating the impact of the different inlet boundary conditions on the solution (in a similar way to Hua et al. [17]), and assessing the hypothesis of nonNewtonian behavior of blood (using the Carreau model), in comparison with the usual approximation of blood as a Newtonian fluid.

\section{Materials and methods}

Signed informed consent was obtained from the patients and all procedures were performed in accordance with the Declaration of Helsinki and submitted to the local institutional medical ethics committee. Two patients with PAA and endovascularly treated at Vascular and Endovascular Surgery Unit of University Hospital of Genoa were enrolled for an imaging study with double CT acquisition at straight- and bent-leg. More details of the imaging acquisition protocol were provided in a previous study [18].

A 78-year old man (Patient 1) with PAA in the left leg was successfully treated with two Viabahn devices (W.L. Gore $\&$ Associates, Flagstaff, AZ, USA) sized $9 \mathrm{~mm} \times 150 \mathrm{~mm}$ (proximal stent) and $7 \mathrm{~mm} \times 150 \mathrm{~mm}$ (distal stent). At 12 months follow-up, post-operative CT showed partial stent thrombosis in the transition zone between the two partially overlapped devices. The second enrolled patient (Patient 2) was a 68 year-old man treated in the left leg for PAA with two Viabahn devices sized $10 \mathrm{~mm} \times 100 \mathrm{~mm}$ (proximal stent) and $9 \mathrm{~mm} \times 150 \mathrm{~mm}$ (distal stent). In this patient, intra-stent thrombosis was revealed already by 1 month follow-up CT scan.

Postoperative CT images were anonymized and transferred to a workstation for image processing. Segmentation 
of the vessel lumen from the femoral artery bifurcation to the popliteal artery bifurcation, intra-stent thrombosis, leg bones, and the implanted stent-graft(s) was performed by means of Vascular Modeling ToolKit (VMTK) libraries [19]. A surrogate pre-thrombotic model of the lumen was derived by virtually removing the thrombosis during the image segmentation in order to correlate hemodynamic with thrombosis onset. Rigid registration of bent-leg structures on their corresponding straight counterparts was automatically performed by means of the Iterative Closest Point algorithm implemented in VMTK. Centerline vessel was automatically extracted, smoothed and resampled by $0.5 \mathrm{~mm}$ by means of VMTK libraries [20]. Centerline tortuosity was then computed: it represents an important factor in different cardiovascular diseases, i.e., atherosclerosis, abdominal aortic aneurysm, and, in particular, in thrombus initiation [21]. Tortuosity $(T)$ is measured as follows: given the centerline length $(L)$ and the shortest distance between the two centerline endpoints $(E D), T=L / E D-1$; therefore, with this definition, the tortuosity of a straight line is 0 .

Transient CFD analyses were performed using Intel Xeon W-2123 computing workstation $(3.6 \mathrm{GHz}, 32 \mathrm{~GB}$ RAM) with the commercial software FLUENT (v.19.2, ANSYS Academic Research). We considered the two patients in both straight- and bent-leg configurations in order to assess the effects of leg bending and the impact of inlet boundary condition on the flow solution. Uniform meshes were generated using VMTK, ranging from 648879 and 1471373 number of tetrahedral elements, according to the previously performed mesh sensitivity analysis. In particular, the mesh was refined until the difference in the luminal area exposed to TAWSS $<0.62 \mathrm{~Pa}$ between successive grids was $<1 \%$. Geometry and boundary conditions are the main features affecting the CFD simulations in hemodynamics. We evaluated the impact of the inlet boundary conditions on the numerical solution by considering two literature waveforms (boundary conditions A and B) with three different scenarios, i.e., with or without flow extension and varying the velocity profile (flat or parabolic). In particular, the flow extensions that we used in the simulations have been chosen in order to reduce the effect of the arbitrary choice of the velocity profile. They were modeled using VMTK, with a length corresponding to 3.5 times the dimension of the inlet diameter, according to Colombo et al. [8]. The following inlet boundary conditions were tested:

- A1: velocity inlet waveform taken from Wood et al. [6] (see Fig. 1) with a flat velocity profile.

- A2: equivalent to the boundary condition A1 with the flat velocity profile set at the flow extension of the inlet of the patient-specific models (see Fig. 2).
- A3: equivalent to the boundary condition A2 with a parabolic velocity profile set at the inlet of the flow extension.

- B1: inflow waveform taken from Nichols et al. [22] (see Fig. 1) with a flat velocity profile.

- B2: equivalent to the boundary condition B1 but with the flat velocity profile set at the inlet of the flow extension.

- B3: equivalent to the boundary condition B2 but with a parabolic velocity profile set at the inlet of the flow extension.

Hence, we are imposing the same velocity waveform when using the boundary conditions A1, A2, and A3, while the same inflow waveform when adopting the boundary conditions B1, B2, and B3, thus implying slightly different inlet velocity waveforms according to the inlet radius of our computational domain. For example, in Fig. 1 the velocity waveforms corresponding to the boundary conditions A1 (equivalent to A2, A3) and B1 for Patient 1 in straight-leg configuration are represented. In particular, we extracted the values of the velocity waveform, taken from Ref. [6] (and analogously for the inflow waveform [22]), using the software WebPlotDigitizer 4.4 (WebPlotDigitizer). Then, the data obtained by the literature waveform were interpolated with an 8th order Fourier series by using the Curve Fitting App, given by the software Matlab R2018a (The Mathworks Inc.). The transient inlet velocity waveform was defined in FLUENT by the meaning of a user defined function (UDF). Therefore, the inlet boundary conditions with a flat velocity profile (i.e., scenarios A1, A2, B1, B2) were given by



Fig. 1 Inlet velocity waveforms in $\mathrm{m} / \mathrm{s}$ colored according to: the literature inlet velocity taken from Wood et al. [6] and imposed at the inlet boundary in scenario A1 (and analogously in A2, A3); the inlet velocity computed from the literature inflow taken from Nichols et al. [15] and used in scenario B1 (and analogously in B2, B3). 


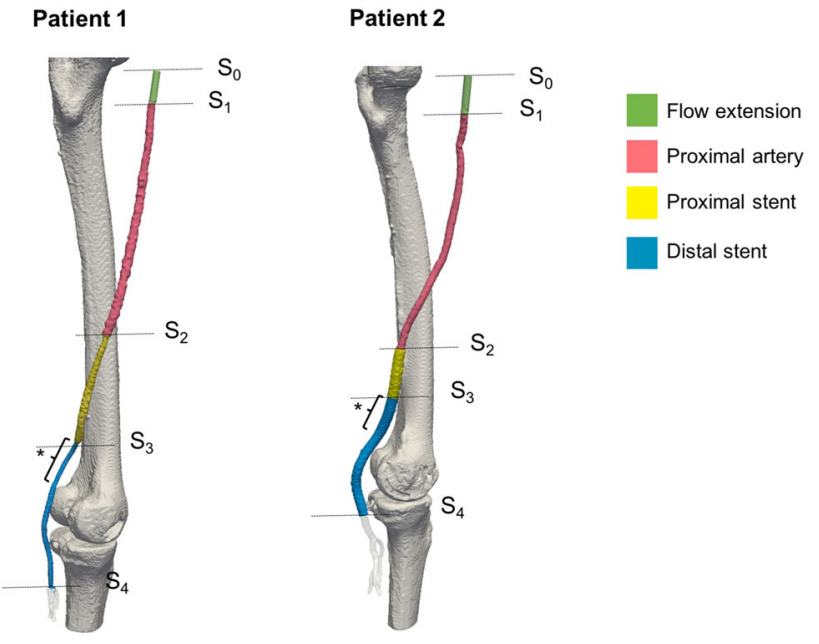

Fig. 2 Femoro-popliteal artery of the two patients considered in the CFD simulations in the straight-leg configuration. Both are colored according to the flow extension, added to our computational domains in scenarios A2, A3, B2, B3, and to the three zones under investigation (proximal artery, proximal stent, and distal stent). Moreover, the sections considered in the post processing $\mathrm{S}_{0}, \mathrm{~S}_{1}, \ldots, \mathrm{S}_{4}$ are represented. The region marked with asterisk denotes the overlapping zone of the two stents in both the patients

$u_{\text {flat }}=a_{0}+\sum_{i=1}^{8}\left[a_{i} \cos (\mathrm{i} \omega t)+b_{i} \sin (\mathrm{i} \omega t)\right]$

where $\omega$ is the fundamental frequency (see Table 1), $t$ the simulation time, and $a_{0}, a_{i}, b_{i}$ for $i=1,2, \cdots, 8$, the values of the Fourier parameters given by the Curve Fitting App. The inlet boundary conditions with a parabolic velocity profile (i.e., scenarios A3 and B3) were prescribed as

$u_{\text {parabolic }}=2 u_{\text {flat }}\left[1-\left(\frac{r}{R}\right)^{2}\right]$,

where $r$ denotes the distance between a point on the constrained surface and the center of the surface, and $R$ is the radius of the constrained surface.

The proposed six boundary conditions were imposed on the patient-specific model of the two patients for both straight- and bent-leg configurations, therefore we performed 24 simulations ( $s i x$ boundary conditions for two patients for two leg configurations).

Firstly, blood was assumed as an incompressible and Newtonian fluid, with $1060 \mathrm{~kg} / \mathrm{m}^{3}$ density and $0.0035 \mathrm{~Pa}$ $\mathrm{s}$ viscosity [16]. Then, in order to evaluate the impact of the non-Newtonian behavior of blood, we chose the boundary conditions A1 and B1, i.e., two velocity waveforms with a flat velocity profile, running 8 simulations (two boundary conditions per two patients per two leg configurations). The viscosity was modeled using the Carreau model described in the following equation: $\eta=\eta_{\infty}+\left(\eta_{0}-\eta_{\infty}\right)\left(1+\lambda^{2} \dot{\gamma}^{2}\right)^{(n-1) / 2}$,

where $\eta$ is the effective viscosity, $\eta_{\infty}$ the infinite shear rate viscosity, $\eta_{0}$ the zero shear rate viscosity, $\lambda$ the natural time, $\dot{\gamma}$ the shear rate, and $n$ the power law index. The parameter values were set according to Quanyu et al. [23] and listed in Table 1. In each simulation we prescribed the no-slip condition on the wall of the artery. Regarding the outlets, the following flow splits were assigned as percentages of the FPA output, according to Crawford et al. [24]: the anterior tibial artery $20 \%$, posterior tibial artery $40 \%$, and peroneal artery $40 \%$. The flow was assumed in laminar regime since the maximum Reynolds number among all the simulations was 1328 at systolic peak (occurring with A1, A2, and A3 conditions). Semi-implicit method for pressure linked equations (SIMPLE) was used to solve the Navier-Stokes equations. Second order scheme for both pressure and momentum spatial discretization was adopted. After a sensitivity analysis, a constant time-step size was set to $0.001 \mathrm{~s}$ and three cardiac cycles were performed for each simulation to guarantee the repeatability of solution.

In order to evaluate the impact leg bending on the local hemodynamics of FPA, with a focus on the stented and thrombotic regions, the FPA segments of both the patients were divided into 3 zones (see Fig. 2): (1) proximal artery, i.e., lumen of the artery above the proximal end of the proximal stent (excluding the flow extension); (2) proximal stent, i.e., the lumen of the proximal stent, excluding the overlapping zone; (3) distal stent, i.e., the lumen of the distal stent including the overlapping zone. We performed both a qualitative and quantitative analysis comparing the results of the two patients in straight- and bent-leg configurations obtained from the CFD simulations. Firstly, to evaluate the impact of boundary conditions, we focused on the velocity streamlines, the vectors of velocity magnitude, and the velocity profiles at the following cross sections corresponding to: the flow extension inlet, $\mathrm{S}_{0}$; FPA inlet, $\mathrm{S}_{1}$; proximal stent inlet, $\mathrm{S}_{2}$; distal stent inlet, $\mathrm{S}_{3}$; distal stent outlet, $\mathrm{S}_{4}$ (see Fig. 2). The velocity streamlines, the vectors of velocity magnitude, and the velocity profiles were reported at the systolic peak.

We computed the time-averaged wall shear stress (TAWSS) and oscillatory shear index (OSI), regarding the near wall flow features, and local normalized helicity (LNH) and helicity intensity ( $h_{2}$ index), relating to the bulk flow. TAWSS and OSI were computed as follow:

$$
\begin{aligned}
& \text { TAWSS }=\frac{1}{T} \int_{0}^{T}|\mathrm{WSS}| \mathrm{d} t \\
& \mathrm{OSI}=0.5\left(1-\frac{\left|\int_{0}^{T} \mathrm{WSS} \mathrm{d} t\right|}{\int_{0}^{T} \mathrm{WSSd} t}\right),
\end{aligned}
$$


Table 1 Fundamental frequency used in UDF inlet waveform—Eq. (1); parameters of the Carreau model—Eq. (3)

\begin{tabular}{llllll}
\hline & Frequency $\omega\left(\mathrm{s}^{-1}\right)$ & $\begin{array}{l}\text { Infinite shear rate } \\
\text { viscosity } \eta_{\infty}(\mathrm{kg} /(\mathrm{m} \cdot \mathrm{s}))\end{array}$ & $\begin{array}{l}\text { Zero shear rate } \\
\text { viscosity } \eta_{0}(\mathrm{~kg} /(\mathrm{m} \cdot \mathrm{s}))\end{array}$ & Time constant $\lambda(\mathrm{s})$ & Power low index $n$ \\
\hline Value & 6.981 & 7.854 & 0.0035 & 0.056 & 3.313 \\
\hline
\end{tabular}
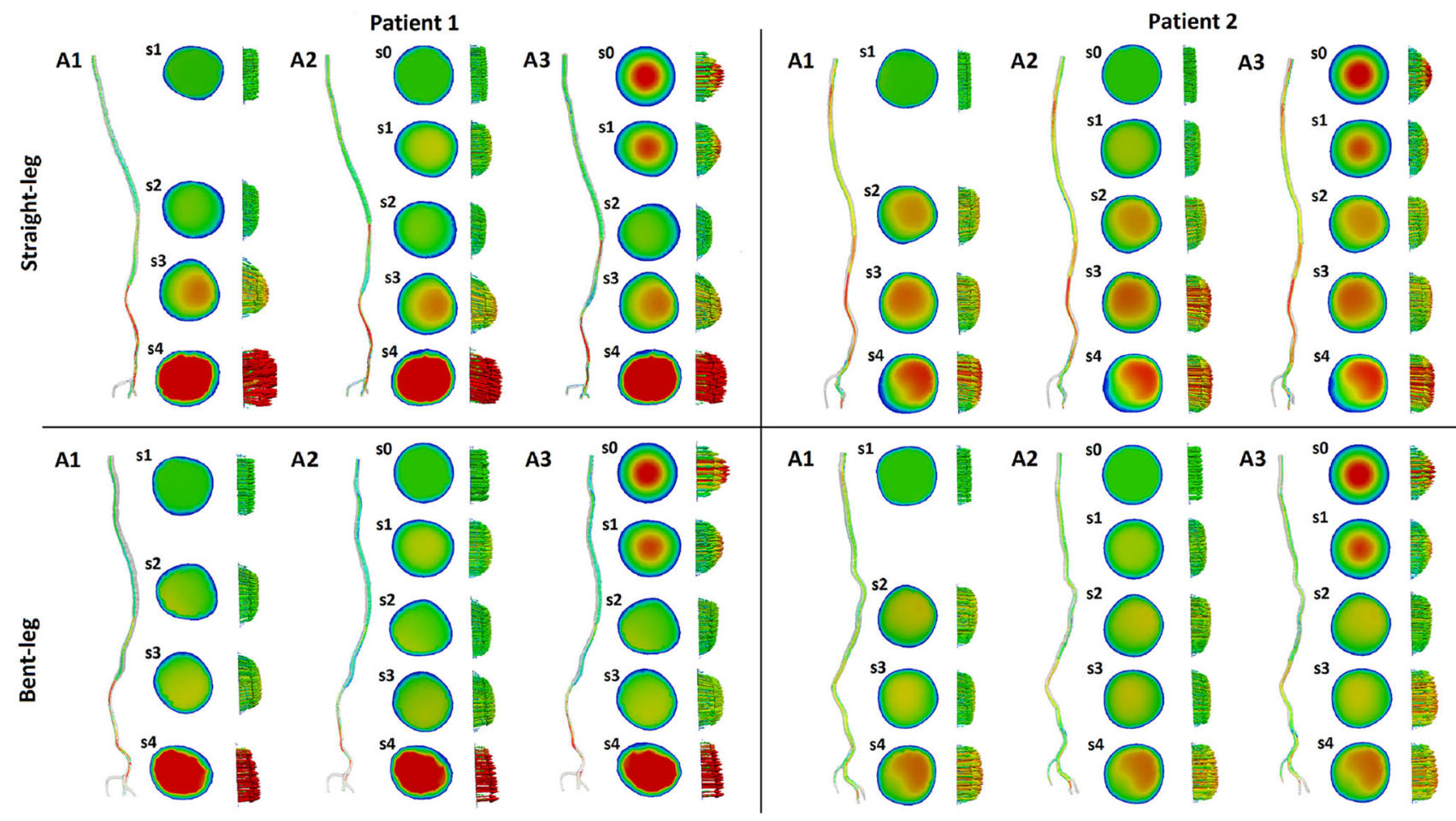

$0.0 e+00 \quad 0.20 .30 .40 .50 .60 .70 .80 .911 .2 e+00$

Fig. 3 Streamlines, contours, and velocity vectors colored according to velocity magnitude at systolic peak, corresponding to the scenarios A1, A2 and $\mathrm{A} 3$, in both straight- and bent-leg configurations of the two patients

where $T$ is the cardiac period and |WSS| the norm of the WSS vector. WSS is defined as follows

$\mathrm{WSS}=\sigma n-[(\sigma n) \cdot n] n$,

where $\sigma$ is the Cauchy stress tensor and $n$ the normal vector to the surface. In particular, in an incompressible fluid, the Cauchy stress tensor is defined as follow

$\sigma=\eta\left(\nabla u+\nabla u^{\mathrm{T}}\right)-p I$,

where $\mathbf{u}$ is the velocity vector, $p$ the pressure, and $\mathbf{I}$ the identity matrix. TAWSS plays a pivotal role in the development of arterial stenosis and in prediction of the risk of wall rupture and thrombus deposition. According to Malek et al. [25], we calculated the luminal surface exposed to low and high values of TAWSS, i.e., ranging between 0 and $0.4 \mathrm{~Pa}$ and above $1.5 \mathrm{~Pa}$, respectively. OSI measures the temporal oscillations of the WSS. In particular, high values of OSI denote sites where the WSS deviates from the main flow direction in a large fraction of the cardiac cycle [26]. According to Gokgol et al. [3], luminal area exposed to high OSI $(>0.3)$ was computed. Regarding the bulk flow, qualitatively, we computed the LNH, which corresponds to the cosine of the angle formed between the vorticity vector and the velocity vector

$\mathrm{LNH}=\frac{(\nabla \times u) \cdot u}{|\nabla \times u| \cdot|u|}=\cos \alpha$,

where $\alpha$ is the angle formed between the vorticity vector $(\nabla \times \mathbf{u})$ and velocity vector $\mathbf{u}$. It is a measure of the alignment/misalignment of the local velocity and vorticity vectors. LNH ranges from -1 to 1 , and its sign indicates the direction of helical structures. Quantitatively, we computed the $h_{2}$ helicity, that is an index regarding the bulk flow: it is given by time-averaging the absolute value of the helicity [27]: 


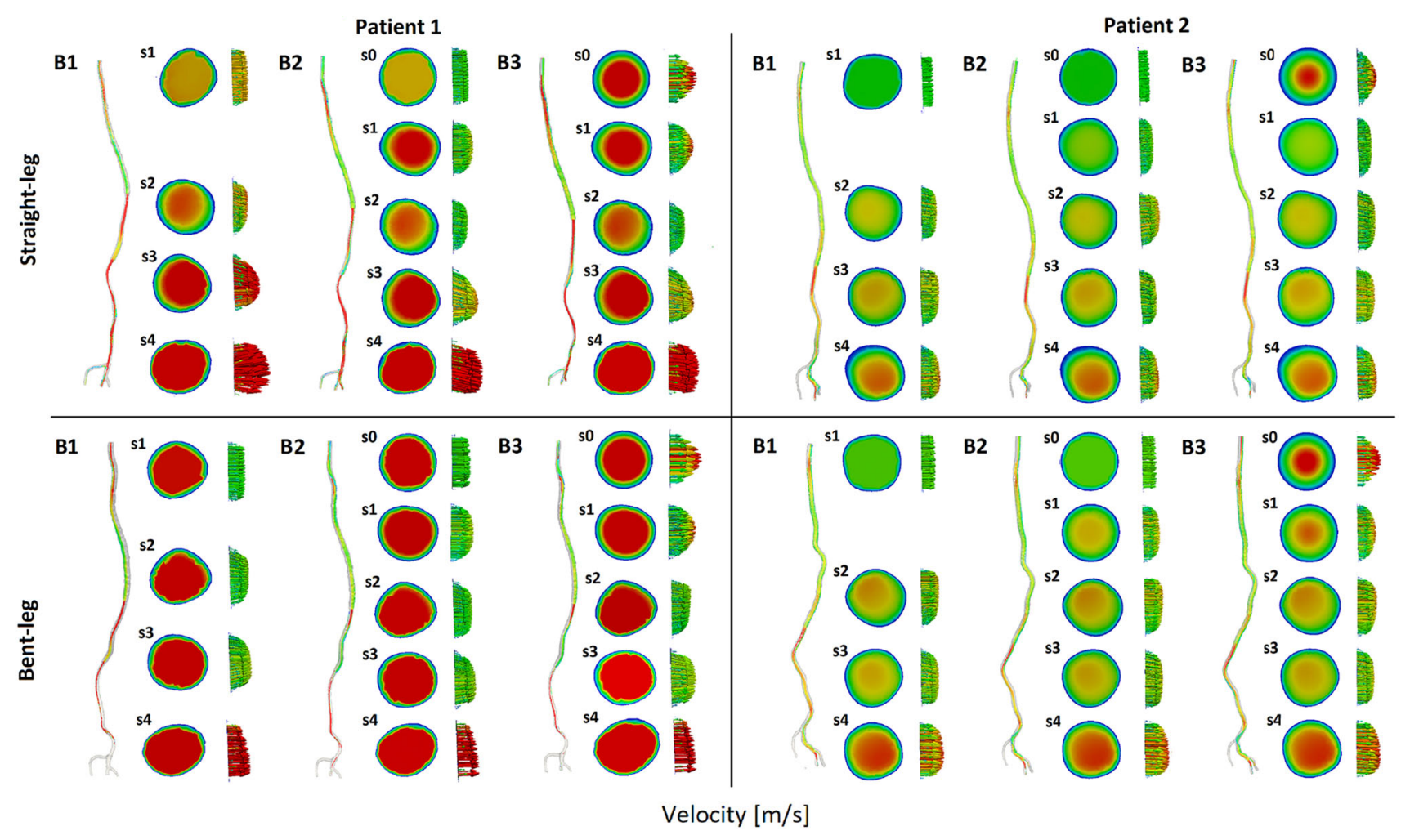

$0.0 e+00 \quad 0.10 \cdot 150 \cdot 20 \cdot 250 \cdot 30.350 .40 .450 .5 \quad 6.0 e-01$

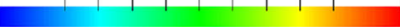

Fig. 4 Streamlines, contours, and velocity vectors colored according to velocity magnitude at systolic peak, corresponding to the scenarios B1, B2, and $\mathrm{B} 3$ in both straight- and bent-leg configurations of the two patients

$h_{2}=\frac{1}{T V} \int_{T} \int_{V}|\mathbf{u} \cdot(\nabla \times \mathbf{u})| \mathrm{d} V \mathrm{~d} t$,

where $V$ is the arterial volume. The $h_{2}$ helicity index expresses the helicity intensity in the fluid domain, irrespective of direction. Recalling that the helicity is defined by the spatial integral of the scalar product of the velocity and vorticity, we assume $h_{2}$ index has higher values in the fluid domain in which velocity and vorticity vectors are aligned.

\section{Results}

Firstly, we reported the results obtained by the 24 simulations performed with constant viscosity. Figures 3 and 4 show the results of CFD simulations for the straight- and bent-leg configurations of both the patients in the six scenarios that have been tested (A1, A2, A3 and B1, B2, B3 in Figs. 3 and 4 , respectively), reporting streamlines, velocity profiles, and velocity vectors colored according to the velocity magnitude at the systolic peak. These two figures prove that only the imposed waveform at the inlet (taken from Wood et al. [6] or Nichols et al. [22]) significantly affects the solution. Indeed, each scenario has similar velocity profiles and contours in the cross-sections of the stented regions, by fixing the inlet waveform. Therefore, from now on we consider only the results relating to the scenarios $\mathrm{A} 1$ and $\mathrm{B} 1$ for both the patients in straight- and bent-leg configurations. However, the figures including all the scenarios relating to the Newtonian model are contained in the Supplementary Materials and Methods section.

Figure 5 highlights the arterial lumen colored according to low (ranging from 0 to $0.4 \mathrm{~Pa}$ ) and high TAWSS (higher than $1.5 \mathrm{~Pa}$ ). The results suggest that the distal part of the artery is exposed to high TAWSS in both straight- and bentleg configuration with a limited influence of inflow boundary conditions; such a result is particularly evident in the case of Patient 1, while for Patient 2 the B1 scenario is resulting in physiological TAWSS in most of the whole artery for both configurations. Figure 6 shows the arterial lumen colored according to high OSI $(>0.3)$ is represented. High OSI are located for all the cases under considerations in the proximal part of artery irrespective to the adopted boundary conditions.

Helical blood flow structures developing into the endoprostheses are represented in Fig. 7 using iso-surfaces of $\mathrm{LNH}$ at the systolic peak with a threshold of \pm 0.25 , according to Colombo et al. [8], for both the patients in straight-leg configuration, relating to the scenario A1. The results show 


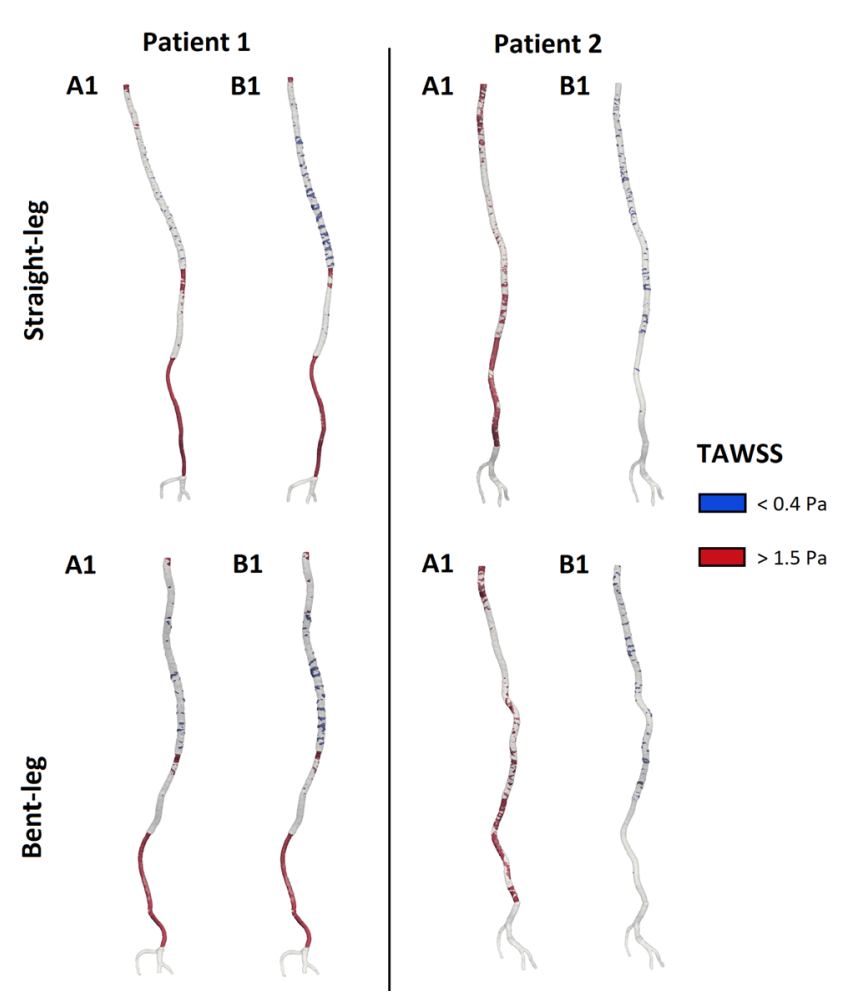

Fig. 5 Arterial lumen colored according to low $(<0.4 \mathrm{~Pa})$ and high $(>$ 1.5 Pa) TAWSS in both straight- and bent-leg configurations of the two patients

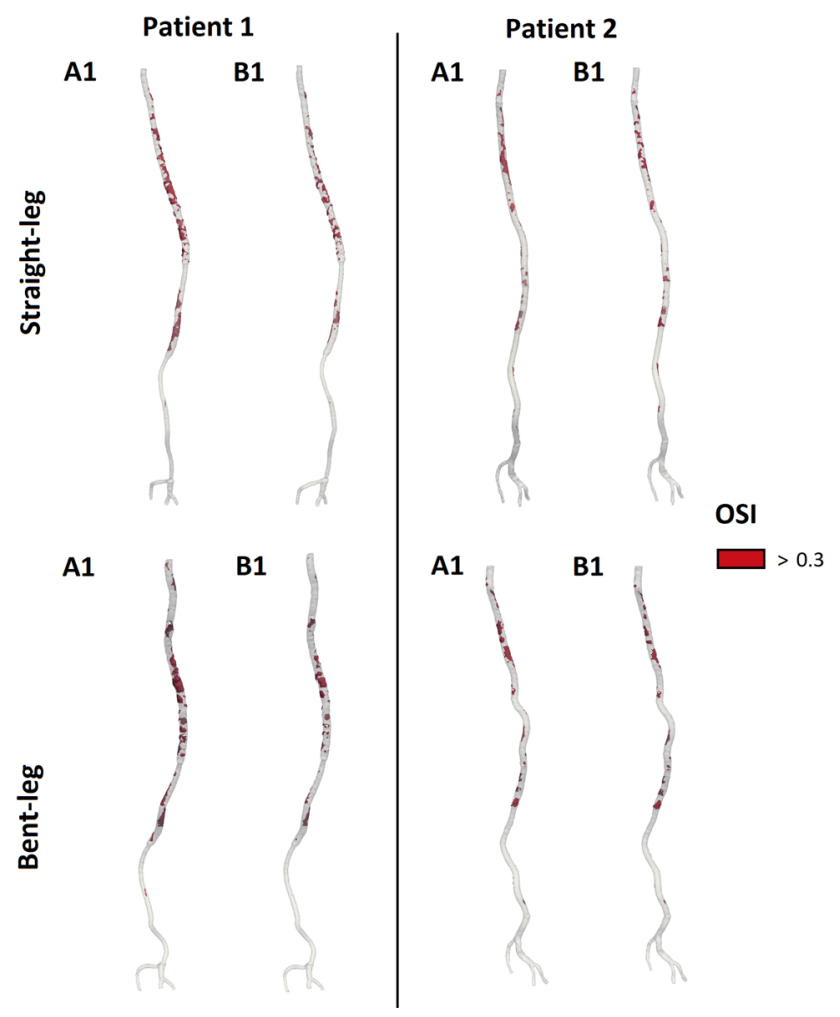

Fig. 6 Arterial lumen colored according to high OSI ( $>0.3)$ in both straight- and bent-leg configurations of the two patients



Fig. 7 Femoro-popliteal artery and three zoom views of the lumen (rotating clockwise) of both the patients in straight-leg configuration: the area where the thrombosis is localized is highlighted by a black box. Moreover, blood flow helicity is represented: in blue the flow with negative $\mathrm{LNH}$ and in red the flow with positive $\mathrm{LNH}$

that the bulk flow in the artery for both patients is characterized by two counter-rotating helical structures and in particular the helical shape of the thrombosis seems to flow the path of the negative LNH region.

Figure 8 reports the bar-plots of the value of $h_{2}$ index, tortuosity, and the percentage of luminal surface exposed to low and high TAWSS, and high OSI corresponding to each zone (proximal artery, proximal stent, and distal stent) for both the patients in straight- and bent-leg configurations. The results show that leg bending induces a difference of the computed hemodynamics indices for Patient 1 with both A1 and B1 boundary conditions. Indeed, a percentage difference above $50 \%$ between the two configurations is present for each hemodynamic quantity that we computed in all the tested scenarios, except for the percentage difference relating to the luminal area exposed to high OSI in Patient 2 (with a maximum percentage difference of $24 \%$ in the distal stent region). In particular, our results show a significant variation of tortuosity between the two configurations, accentuated in the distal stent zone, where the tortuosity is greater in the bent-leg configuration.

Finally, we treated the blood as a non-Newtonian fluid and we assessed the results, comparing them with the previous analyses, obtained using the Newtonian model. Figure 9 shows the arterial lumen of both patients colored according to TAWSS magnitude, low (ranging from 0 to $0.4 \mathrm{~Pa}$ ) and high TAWSS (higher than 1.5 Pa), based on both the Carreau (non-Newtonian) and Newtonian models. Figure 9 represents only the results relating to the scenario $\mathrm{B} 1$, which provides greater differences between the two models under considera- 
Patient 1

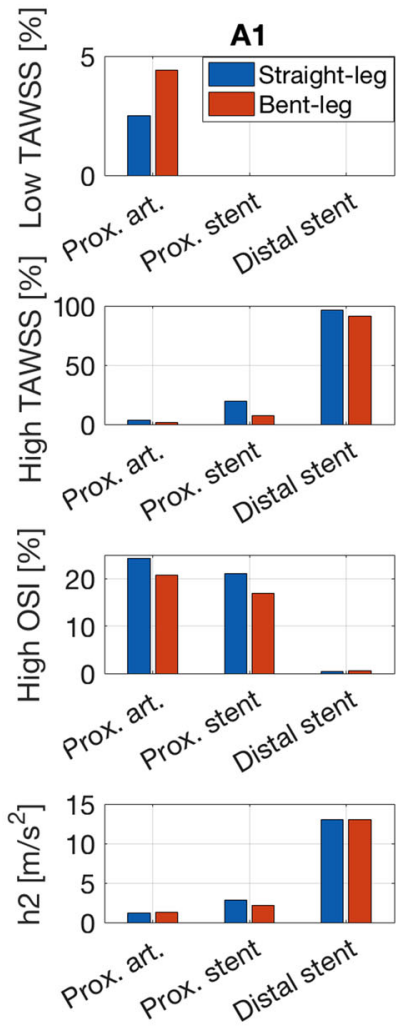

B1




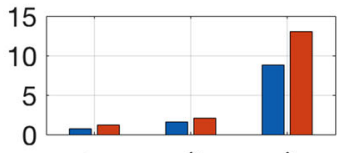

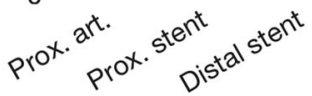

Patient 2

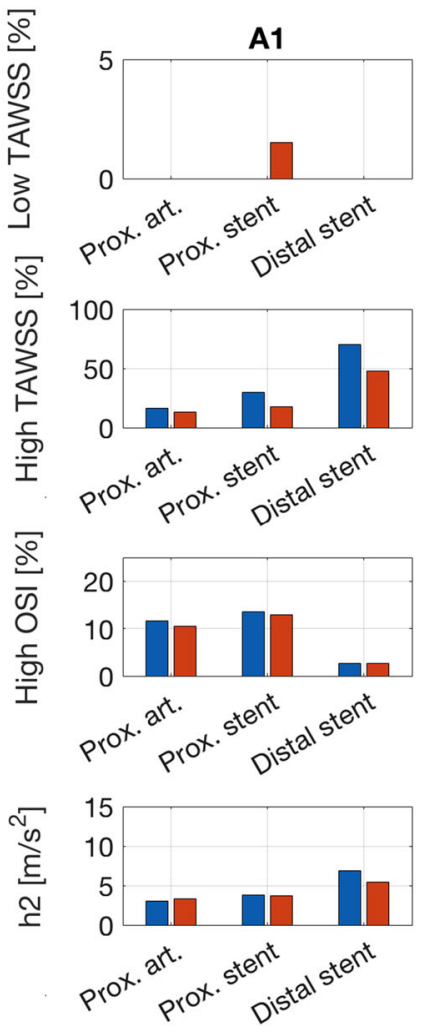

B1
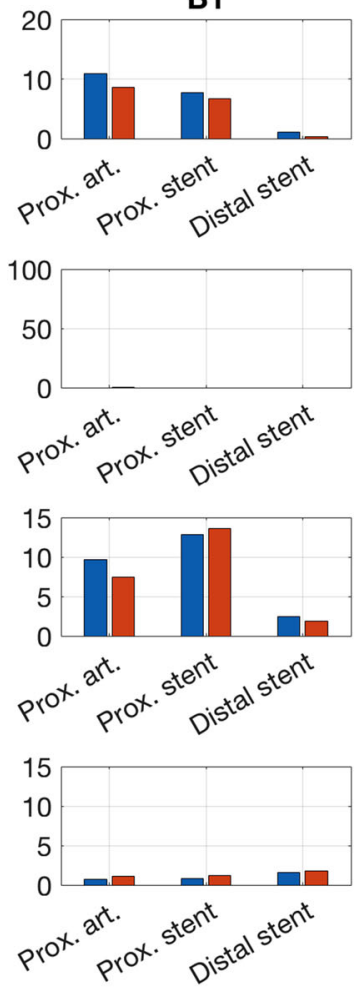

\section{Tortuosity}


Fig. 8 Bar plot of tortuosity, helicity $\left(h_{2}\right.$ index), and percentage of luminal area exposed to both low $(<0.4 \mathrm{~Pa})$ and high $(>1.5 \mathrm{~Pa})$ TAWSS, respectively, and high OSI $(>0.3)$. The data are reported for the three zones under investigation (proximal artery, proximal stent, and distal stent) of the two patients in both leg configurations, corresponding to scenarios A1 and B1

tion and allows us a wider discussion, as we will introduce in the next section. Figures 10 and 11 represent the bar-plots of the value of $h_{2}$ index, and the percentage of luminal surface exposed to low and high TAWSS, and high OSI corresponding to each zone (proximal artery, proximal stent, and distal stent) for both the patients. In particular, Fig. 10 refers to the straight-leg configuration, while Fig. 11 to the bent-leg one.

\section{Discussions}

In this study, we have evaluated the local hemodynamic and the interplay among geometric features in two patients endovascularly treated for PAA, who experienced intra-stent thrombosis during follow-up. In particular, the role of leg bending on the local hemodynamic was elucidated by modeling both straight- and bent-leg configurations.

Focusing on the velocity magnitude, Figs. 3 and 4 show a higher flow velocity in the distal stent region than to the proximal one, due to the luminal narrowing given by the overlapping of the two stents-grafts. As we already pointed out, the results suggest that the different inlet velocity profiles used in the simulations slightly affect the numerical solution, conversely to the determinant role of the prescribed inlet waveform. In order to obtain reliable results of clinical significance, patient-specific inflow waveforms would be very useful in understanding the hemodynamic behavior. However, our geometrical study shows velocity sensitivity, i.e., 
velocity magnitude variations between the two patients occur along the two FPAs by fixing a velocity inlet (i.e., scenario A or B). Although the behavior of stented FPAs has already been investigated in the literature [8], to date there is still no information on the different response between the various portions of the stented artery itself. Figure 8 suggests that the overlapping of the stent grafts seems to induce a severe discontinuity of lumen diameter, dividing the region treated with endovascular stent-graft in two zones: (1) the proximal part, where thrombosis is located, it is characterized by low tortuosity, low velocity, low helicity, low TAWSS, and high OSI; (2) the distal part that presents higher tortuosity, promoting higher velocity, higher helicity, higher TAWSS, and lower OSI. In particular, by focusing on the tortuosity of the vessel (see Fig. 8 at the bottom), the stented FPA respects the behavior that we would have expected, when considered in its entirety, i.e., increased tortuosity values with leg bending. Analyzing the stented area by portions, we have found that in both patients the tortuosity increases from the proximal artery region to the distal one; this result matches the findings of Wood et al. [6], who performed CFD simulations in the superficial femoral artery of 9 healthy men and 9 healthy women, showing that tortuosity was significantly greater for men than women, but the highest values were found in the most distal segment, regardless of sex. Then, when considering the comparison between straight- and bentleg configuration, we observed that in both patients proximal vessel and distal stent segments tortuosity increases with leg bending. However, the proximal stent, characterized by its larger diameter, slow velocity, low TAWSS, and low helicity, straightens with leg flexion. This area is also the one in which thrombosis was found in both patients, confirming that the formation of thrombosis is linked to a combination of both hemodynamic and geometric factors. Hence the importance of conducting the analyses by investigating the stented FPA not only in its entirety but by dividing it into the various portions, in order to be able to identify areas more at risk of thrombosis.

The role of low TAWSS in thrombotic regions has been previously corroborated in literature. Boyd et al. [28] showed a correlation between regions of low WSS, where flow recirculation predominated, and thrombus deposition, by performing CFD simulations in 7 abdominal aortic aneurysms. The luminal area exposed to low TAWSS and high OSI in the proximal zone is greater in Patient 1 than in Patient 2 (see Fig. 8), suggesting that patient-specific geometrical features also affect the near wall flow features.

Regarding the bulk flow, our results suggest that intrastent thrombosis is located in the region where the intensity of helicity is low (see Fig. 8). The fundamental role of helical (or swirling flow) in the prevention of thrombosis and disease progression has been confirmed in many literature studies [29-31]. In particular, Morbiducci et al. [30] also presented

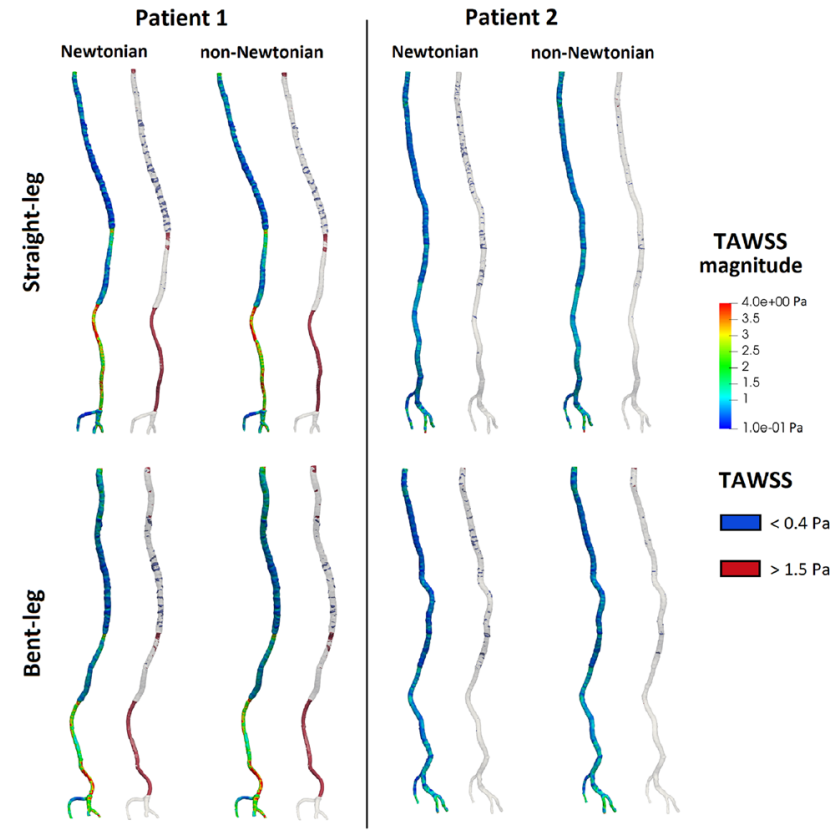

Fig. 9 Arterial lumen colored according to the TAWSS magnitude, low $(<0.4 \mathrm{~Pa})$ and high TAWSS $(>1.5 \mathrm{~Pa})$ in both straight- and bent-leg configurations of the two patients. The TAWSS values represented refers to the scenario B1

an inverse relationship between helical flow and OSI evaluating four bypass geometries in ascending aorta, according to our results. Moreover, our results denote that the spiral shape of thrombosis matches the path of the negative LNH region; this is more evident in Patient 1 (Fig. 7). Figure 7 refers only to scenario A1, but an analogous pattern of the LNH was found using the boundary condition B1. It is hard to formulate a conclusion to explain this result; given the limited number of analysed patients, further analysis involving a cohort of patients should be investigated in order to provide more information to elucidate this observation.

From our results we found that the study in both straightand bent-leg configurations is crucial in understanding and assessing the numerical results in stented arteries, given the increase of the tortuosity of the distal part of the artery due to the leg bending. Our findings match with Wensing et al. [32], who highlighted the importance of considering the impact of knee flexion in femoral and popliteal arteries, showing increasing tortuosity in bent-leg configuration of 22 healthy volunteers. Moreover, the increase of tortuosity in leg bending implies a reduction of the blood velocity in each scenario that we assumed for both patients (see velocity streamlines and contours represented in Figs. 3 and 4).

The alternate bending of the legs is known to influence the mechanical solicitation of the stent [33], the shape of the artery [34], and the local hemodynamics [9] but its role in the thrombosis onset and progression is still unknown. From our results, it is evident that leg bending increases the tortuosity 
Scenario A1

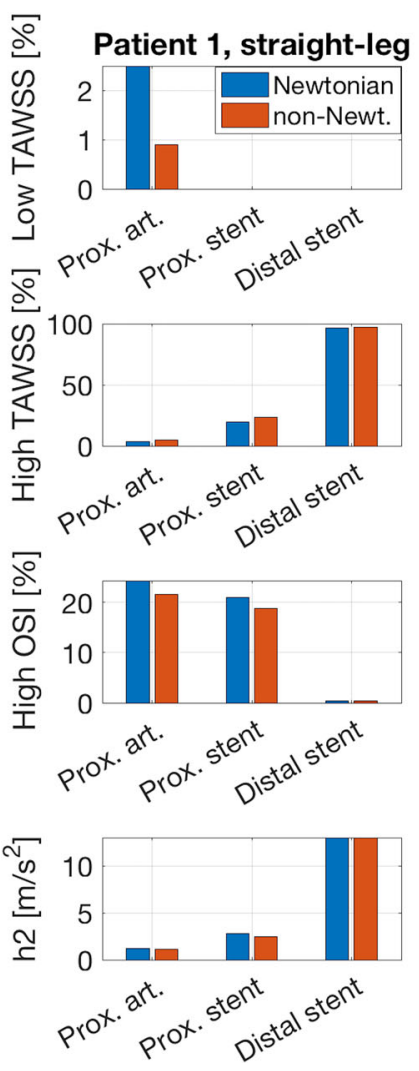

Scenario B1
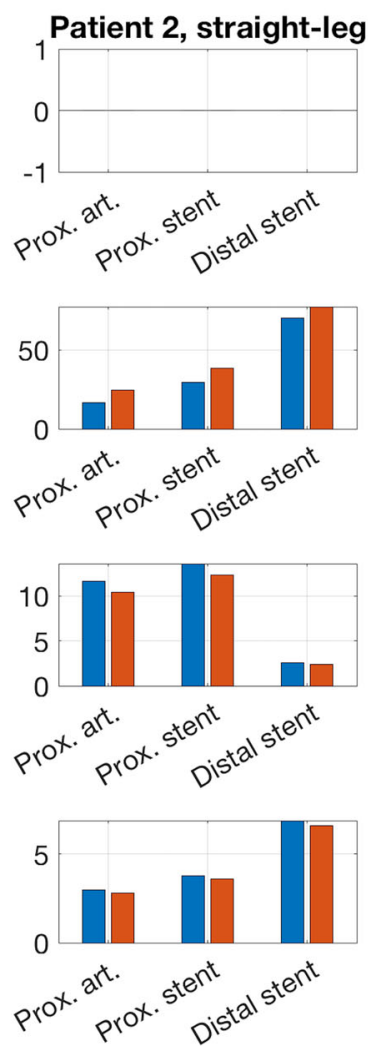
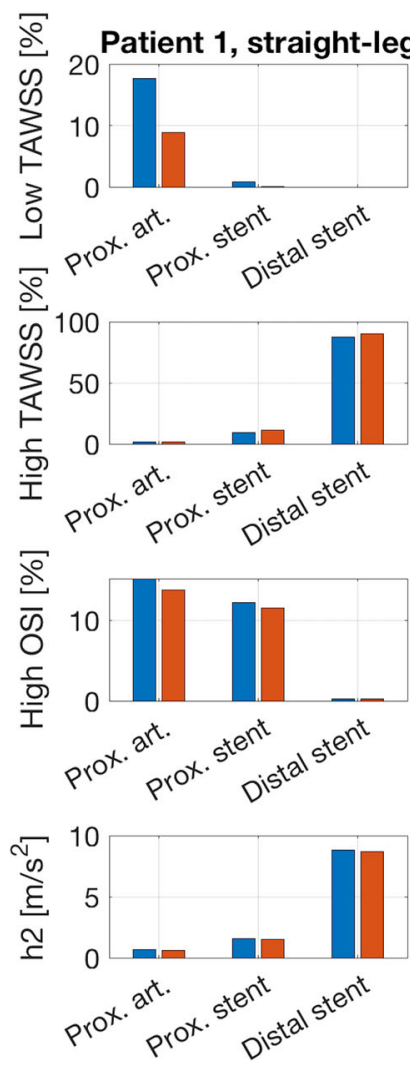
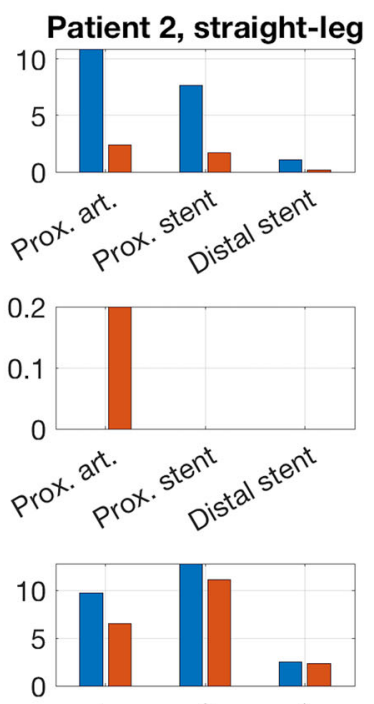



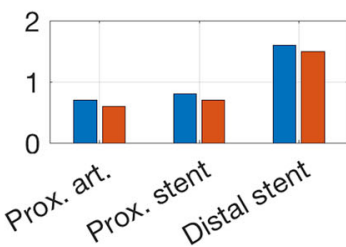

Fig. 10 Comparison between results considering Newtonian and non-Newtonian behavior: bar plot of helicity ( $h_{2}$ index), and percentage of luminal area exposed to both low $(<0.4 \mathrm{~Pa})$ and high $(>1.5 \mathrm{~Pa})$ TAWSS, respectively, and high OSI $(>0.3)$. The data are reported for the three zones under investigation (proximal artery, proximal stent, and distal stent) of the two patients in the straight-leg configuration, corresponding to scenarios A1 and B1

of the distal stent segment, which combined with an overall blood flow velocity, exacerbate the difference between the distal and proximal part of the stented region, with the latter more exposed to the risk of thrombosis (i.e., lower velocity, wider area of low wall shear stress, higher oscillatory shear stress, and lower helicity). Such considerations are however hardly generalizable with data proposed by the present paper, which deals with only two patients, but, at the same time, call for future developments focused on such hypotheses.

Focusing on the qualitative comparison between the Newtonian and non-Newtonian model, Fig. 9 shows an optimal agreement on the distribution of the TAWSS magnitude between the two models. These results reproduce the assumptions discussed by Liu et al. [35], who introduced that the blood viscosity properties do not affect the spatial pattern of the TAWSS qualitatively. However, looking at the luminal surface exposed to low and high TAWSS, the area with low TAWSS is greater in the Newtonian model for both the patients, while no significant difference occurs between the surfaces with high TAWSS. Our findings are in agreement with Soulis et al. [15] and Liu et al. [35], who proved an underestimated WSS given by the Newtonian model, when the magnitude of WSS is relatively small $\left(<1 \mathrm{~N} / \mathrm{m}^{2}\right)$. Analogously, we found similar results using the scenario A1, but with less marked differences, since the surface exposed to low TAWSS is very small even in the Newtonian model. For this reason we chose to omit the qualitative analysis given by the scenario A1.

Figures 10 and 11 allow deepening the comparison between the Newtonian and non-Newtonian models. Significant differences based on the luminal surfaces exposed to low TAWSS are highlighted (with a maximum decrease in the proximal artery zone, compared to the non-Newtonian model, of $8.7 \%$ and $8.5 \%$ for Patients 1 and 2, respectively, in the straight-leg configuration and in the scenario B1), a good agreement occurs for the other analyzed outcomes (with a maximum OSI decrease in the proximal artery zone, compared to the non-Newtonian model, of $3.2 \%$ for patient 2 in the straight-leg configuration and in the scenario B1). In particular, as we mentioned before, the scenario B1, in which the inlet average velocity is lower and also low TAWSS values occur, provides major differences. As the velocity increases (see the results given by the scenario A1 in Fig. 10), the 


\section{Scenario A1}

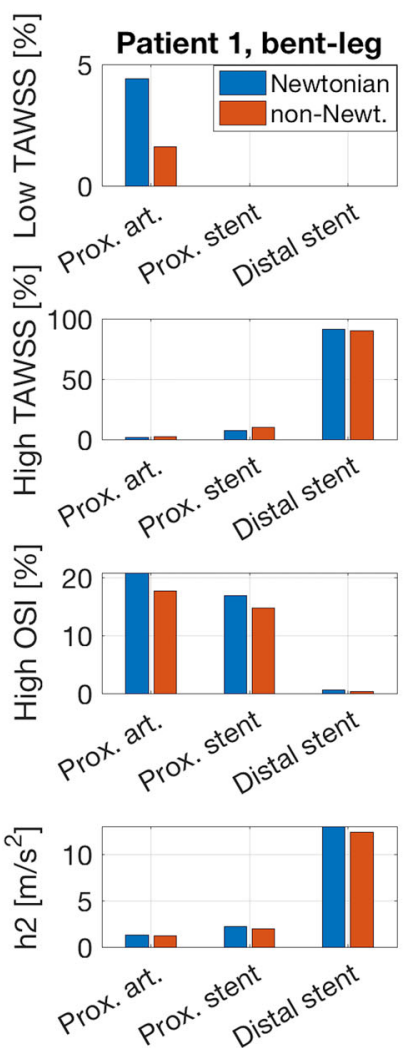

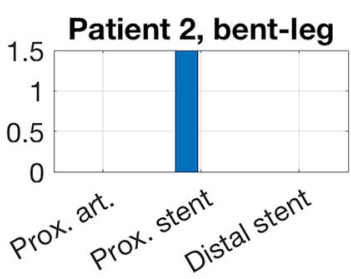
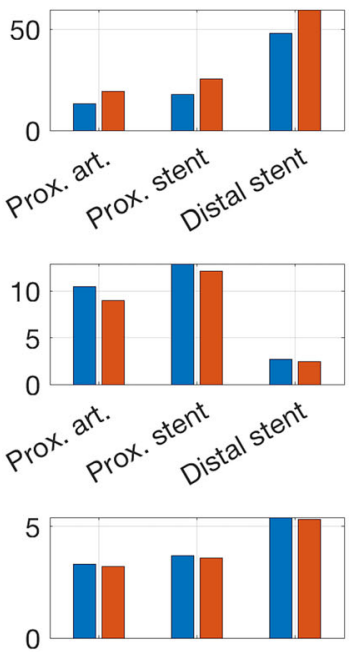

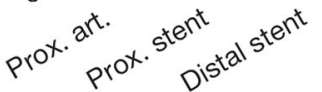

Scenario B1
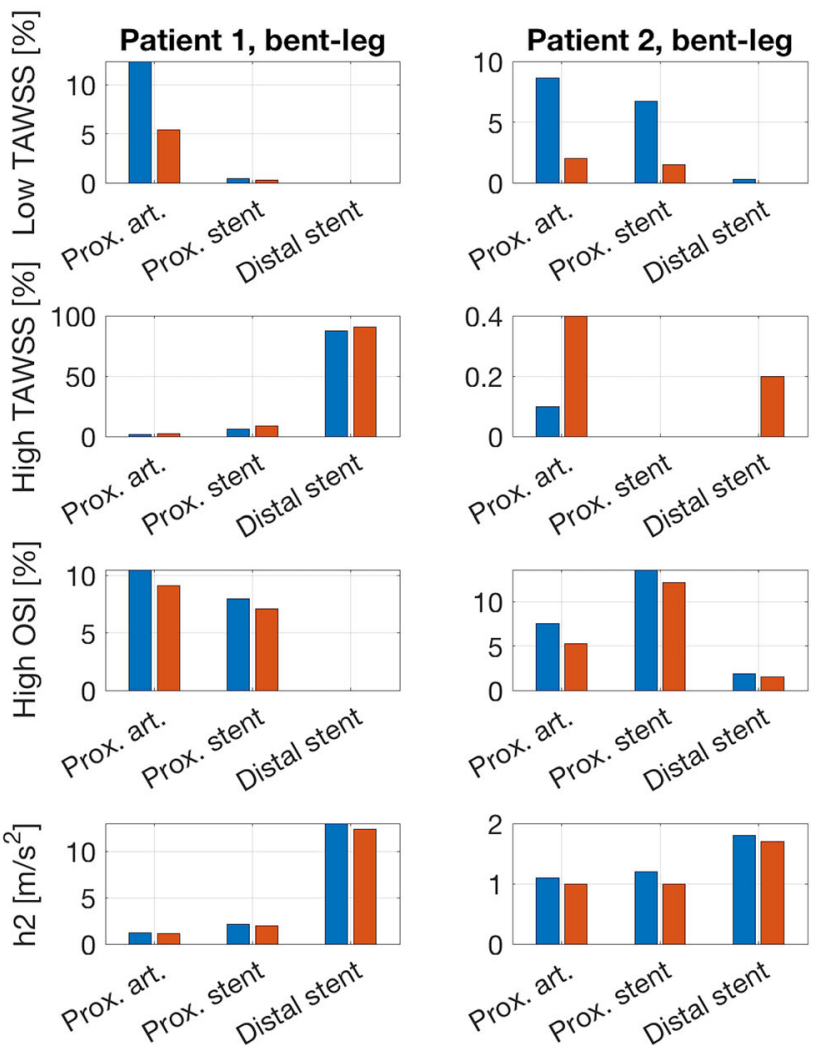

Fig. 11 Comparison between results considering Newtonian and non-Newtonian behavior: bar plot of tortuosity, helicity ( $h_{2}$ index), and percentage of luminal area exposed to both low $(<0.4 \mathrm{~Pa})$ and high $(>1.5 \mathrm{~Pa})$ TAWSS, respectively, and high OSI $(>0.3)$. The data are reported for the three zones under investigation (proximal artery, proximal stent, and distal stent) of the two patients in the bent-leg configuration, corresponding to scenarios A1 and B1

Newtonian and non-Newtonian models become more similar, according to Liu et al. [35].

\section{Limitations}

The present work, based on the analysis of only two cases, represents a proof-of-concept study, aimed at linking post-stent geometry, hemodynamics, and thrombosis in endovascular repair of popliteal aneurysms. Further analyzes should be performed in order to obtain statistically and clinically relevant conclusions. We have already discussed the importance of considering patient-specific inlet boundary condition; therefore, in future studies inflow data elaborated by echo doppler measurements will be set at the inlet of the computational domain.

The computational domains considered in the simulations represent surrogate geometrical models of the lumen of each patient prior to thrombosis by virtually removing the thrombus during the segmentation process. Such a limitation could be overcome by analyzing the CT scans performed at different time instants, from early post-operative to annual follow-up exams.

In the present study we dealt with thrombosis only from a fluid dynamic point of view. However, further analysis should include the role of hemodynamic stress in the platelet activation [36], recently proved to be associated with aortic thrombus formation [37].

Finally, according to previous studies [3,8], we did not take into account the stent struts; however, further developments will include this aspect, since Al-Hakim et al. [38] showed that stent struts have an effect on WSS.

\section{Conclusions}

The present study suggests that the overlapping of the stentgrafts seems to induce a severe discontinuity of lumen diameter, dividing the region treated with endovascular stentgraft into two zones: the proximal part, where thrombosis is located, it is characterized by low tortuosity, low velocity, low helicity, low TAWSS, and high OSI; the distal part that presents higher tortuosity, promoting higher velocity, higher 
helicity, higher TAWSS, and lower OSI. Since this analysis is limited to two cases, a further study with a cohort of patients should be investigated in order to generalize and validate our results. Boundary conditions affect the solution only when considering different velocity waveforms, dependent on time; different inlet velocity profiles and the use of flow extension do not provide significant variations. Accounting for actual flow rate is essential for accurate and reliable results. The Newtonian and non-Newtonian blood treatments provide similar results in both the patients, except when the magnitude of the TAWSS is relatively small $(<0.4$ $\mathrm{Pa}$ ). In this latter case the Newtonian model gives lower values of TAWSS than the non-Newtonian one. However, the Newtonian blood treatment should be a good choice in all cases in which the analysis of WSS is not necessary. Leg bending induces significant hemodynamic differences compared to the straight leg configuration in each of the scenarios we studied for both patients. The helical form of intra-stent thrombosis suggests an implication of flow helicity in the onset and progression of thrombosis. However, further studies should be considered to investigate this aspect.

Acknowledgements This work was partially supported by the "Programma Operativo Por FSE Regione Liguria 2014-2020” (RLOF18ASSRIC/38/1).

Funding Open access funding provided by Università degli Studi di Pavia within the CRUI-CARE Agreement.

\section{Compliance with ethical standards}

Ethical approval The study was approved by the Liguria Regional Ethics Committee (Comitato Etico Regionale Liguria) on 15/07/2019 (Ref. gr-2018-12368376; internal Amendment No: 4587).

Open Access This article is licensed under a Creative Commons Attribution 4.0 International License, which permits use, sharing, adaptation, distribution and reproduction in any medium or format, as long as you give appropriate credit to the original author(s) and the source, provide a link to the Creative Commons licence, and indicate if changes were made. The images or other third party material in this article are included in the article's Creative Commons licence, unless indicated otherwise in a credit line to the material. If material is not included in the article's Creative Commons licence and your intended use is not permitted by statutory regulation or exceeds the permitted use, you will need to obtain permission directly from the copyright holder. To view a copy of this licence, visit http://creativecomm ons.org/licenses/by/4.0/.

\section{References}

1. Tielliu, I.F., Zeebregts, C.J., Vourliotakis, G., et al.: Stent fractures in the Hemobahn/Viabahn stent graft after endovascular popliteal aneurysm repair. J. Vasc. Surg. 51(6), 1413-1418 (2010)

2. Smouse, H.B., Nikanorov, A., LaFlash, D.: Biomechanical forces in the femoropopliteal arterial segment. Endovasc. Today 4(6), 6066 (2005)
3. Gökgöl, C., Diehm, N., Räber, L., et al.: Prediction of restenosis based on hemodynamical markers in revascularized femoro-popliteal arteries during leg flexion. Biomech. Model. Mechanobiol. 18, 1883-1893 (2019)

4. Glagov, S., Zarins, C., Giddens, D.P., et al.: Hemodynamics and atherosclerosis. Insights and perspectives gained from studies of human arteries. Arch. Pathol. Lab. Med. 112(10), 1018-1031 (1988)

5. Casa, L.D., Deaton, D.H., Ku, D.N.: Role of high shear rate in thrombosis. J. Vasc. Surg. 61(4), 1068-1080 (2015)

6. Wood, N.B., Zhao, S.Z., Zambanini, A., et al.: Curvature and tortuosity of the superficial femoral artery: a possible risk factor for peripheral arterial disease. J. Appl. Physiol. 101(5), 1412-1418 (2006)

7. Desyatova, A., Poulson, W., Deegan, P., et al.: Limb flexion induced twist and associated intramural stresses in the human femoropopliteal artery. J. R. Soc. Interface 14, 20170025 (2017)

8. Colombo, M., Bologna, M., Garbey, M., et al.: Computing patientspecific hemodynamics in stented femoral artery models obtained from computed tomography using a validated 3D reconstruction method. Med. Eng. Phys. 75, 23-35 (2020)

9. Colombo, M., Luraghi, G., Cestariolo, L., et al.: Impact of lower limb movement on the hemodynamics of femoropopliteal arteries: a computational study. Med. Eng. Phys. 81, 105-117 (2020)

10. Conti, M., Ferrarini, A., Finotello, A., et al.: Patient-specific computational fluid dynamics of femoro-popliteal stent-graft thrombosis. Med. Eng. Phys. 86, 57-64 (2020)

11. Merrill, E.W.: Rheology of blood. Physiol. Rev. 49, 863-888 (1969)

12. Wootton, D.M., Ku, D.N.: Fluid mechanics of vascular systems, diseases, and thrombosis. Annu. Rev. Biomed. Eng. 1, 299-329 (1999)

13. Cho, Y.I., Kensey, K.R.: Effects of the non-Newtonian viscosity of blood on flows in a diseased arterial vessel. Part 1: Steady flows. Biorheology 28, 241-262 (1991)

14. Johnston, B.M., Johnston, P.R., Corney, S., et al.: Non-Newtonian blood flow in human right coronary arteries: transient simulations. J. Biomech. 39, 1116-1128 (2005)

15. Soulis, J.V., Giannoglou, G.D., Chatzizisis, Y.S., et al.: Spatial and phasic oscillation of non-Newtonian wall shear stress in human left coronary artery bifurcation: an insight to atherogenesis. Coron. Artery Dis. 17, 351-358 (2006)

16. Desyatova, A., MacTaggart, J., Romarowski, R., et al.: Effect of aging on mechanical stresses, deformations, and hemodynamics in human femoropopliteal artery due to limb flexion. Biomech. Model. Mechanobiol. 17, 181-189 (2018)

17. Hua, Y., Oh, J.H., Kim, Y.B.: Influence of parent artery segmentation and boundary conditions on hemodynamic characteristics of intracranial aneurysms. Yonsei Med. J. 56, 1328-1337 (2015)

18. Spinella, G., Finotello, A., Pane, B., et al.: In vivo morphological changes of the femoropopliteal arteries due to knee flexion after endovascular treatment of popliteal aneurysm. J. Endovasc. Ther. 26(4), 496-504 (2019)

19. Antiga, L., Piccinelli, M., Botti, L., et al.: An image-based modeling framework for patient-specific computational hemodynamics. Med. Biol. Eng. Comput. 46(11), 1097 (2008)

20. Piccinelli, M., Veneziani, A., Steinman, D.A., et al.: A framework for geometric analysis of vascular structures: application to cerebral aneurysms. IEEE Trans. Med. Imaging 28(8), 1141-1155 (2009)

21. Chesnutt, J.K.W., Han, H.-C.: Tortuosity triggers platelet activation and thrombus formation in microvessels. J. Biomech. Eng. 133, 121004-1 (2011). https://doi.org/10.1115/1.4005478

22. Nichols, W., O'Rourke, M.F., Vlachopoulos, C.: McDonald's Blood Flow in Arteries, 6th edn. Hodder Arnold, London (2011)

23. Quanyu, W., Xiaojie, L., Lingjiao, P., et al.: Simulation analysis of blood flow in arteries of the human arm. Biomed. Eng. 29, 1750031-8 (2017) 
24. Crawford, J.D., Robbins, N.G., Harry, L.A., et al.: Characterization of tibial velocities by duplex ultrasound in severe peripheral arterial disease and controls. J. Vasc. Surg. 63, 646-651 (2016)

25. Malek, A.M., Alper, S.L., Izumo, S.: Hemodynamic shear stress and its role in atherosclerosis. J. Am. Med. Assoc. 282, 2035-2042 (1999)

26. Morbiducci, U., Gallo, D., Massai, D., et al.: Outflow conditions for image-based hemodynamic models of the carotid bifurcation: implications for indicators of abnormal flow. J. Biomech. Eng. 132, 091005-1 (2010)

27. Morbiducci, U., Ponzini, R., Gallo, D., et al.: Inflow boundary conditions for image-based computational hemodynamics: Impact of idealized versus measured velocity profiles in the human aorta. J. Biomech. 46, 102-109 (2013)

28. Boyd, A.J., Kuhn, D.C.S., Lozowy, R.J., et al.: Low wall shear stress predominates at sites of abdominal aortic aneurysm rupture. J. Vasc. Surg. 63, 1613-1619 (2016)

29. Caro, C., Watkins, N., Sherwin, S.: Helical graft. Patent US 2007/0021707 A1 (2007)

30. Morbiducci, U., Ponzini, R., Grigioni, M., et al.: Helical flow as fluid dynamic signature for atherogenesis risk in aortocoronary bypass. A numeric study. J. Biomech. 40, 519-34 (2007)

31. Qiu, Y., Yuan, D., Wang, Y., et al.: Hemodynamic investigation of a patient-specific abdominal aortic aneurysm with iliac artery tortuosity. Comput. Methods Biomech. Biomed. Eng. 21, 824-833 (2018)

32. Wensing, P.J.W., Scholten, F.G., Buijs, P.C., et al.: Arterial tortuosity in the femoropopliteal region during knee flexion: a magnetic resonance angiographic study. J. Anat. 186, 133-139 (1995)
33. Conti, M., Marconi, M., Campanile, G., et al.: Patient-specific finite element analysis of popliteal stenting. Meccanica 52, 633-644 (2017)

34. Spinella, G., Finotello, A., Pane, B., et al.: In vivo morphological changes of the femoropopliteal arteries due to knee flexion after endovascular treatment of popliteal aneurysm. J. Endovasc. Ther. 43, 1-9 (2019)

35. Liu, B., Tang, D.: Influence of non-Newtonian properties of blood on the wall shear stress in human atherosclerotic right coronary arteries. Mol. Cell Biomech. 8, 73-90 (2011)

36. Shadden, S.C., Hendabadi, S.: Potential fluid mechanic pathways of platelet activation. Biomech. Model. Mechanobiol. 12(3), 467474 (2013)

37. Nauta, F.J., Lau, K.D., Arthurs, C.J., et al.: Computational fluid dynamics and aortic thrombus formation following thoracic endovascular aortic repair. Ann. Thorac. Surg. 103, 1914-1921 (2017)

38. Al-Hakim, R., Lee, E.W., Kee, S.T., et al.: Hemodynamic analysis of edge stenosis in peripheral artery stent grafts. Diagn. Interv. Imaging 98, 729-735 (2017)

Publisher's Note Springer Nature remains neutral with regard to jurisdictional claims in published maps and institutional affiliations. 